UDK: 811.134.2(73)

DOI: https://doi.org/10.18485/legado_hispanico.2020.ch20

\author{
Gabriela Vokić ${ }^{1}$ \\ Universidad Metodista del Sur \\ Dallas, Tejas, EE.UU.
}

\title{
HABLANTES DE HERENCIA HISPANA EN PROGRAMAS DE LENGUA UNIVERSITARIOS EN LOS EE.UU.: UN PANORAMA SINCRÓNICO
}

\begin{abstract}
Resumen
El propósito de este trabajo es ofrecer una vista panorámica sobre la realidad que enfrenta el estudiante universitario de herencia hispana en los EE.UU. en programas de lengua. Según las proyecciones demográficas más recientes, en un futuro próximo el estudiante de herencia hispana dejará de ser la minoría en el ambiente universitario en los EE.UU. Sin embargo, todavía no existe una metodología acordada para enseñar el español a los hablantes de herencia hispana, pese a que el inglés y el español han coexistido durante siglos en esta región geográfica y que las investigaciones sobre la variedad estadounidense del español recalcan la necesidad de métodos de enseñanza particularmente diseñados para los hablantes de herencia. Partiendo de la literatura existente, este trabajo se dedica a resumir los desafíos que esta discordancia representa para los estudiantes de herencia hispana matriculados en programas de lengua universitarios en la actualidad y contempla algunas soluciones.
\end{abstract}

Palabras clave: hablantes de herencia hispana, programas de lengua española, conciencia lingüística crítica, bilingüismo aditivo, pedagogía crítica.

${ }^{1}$ vokic@smu.edu 


\section{Introducción}

El perfil del estudiante universitario prototípico en los EE.UU. está cambiando a un ritmo veloz gracias a la presencia más marcada de los hablantes de herencia hispana ( $\mathrm{HHH}$ ). En programas de lengua también, el estudiantado es más diverso que nunca, consistiendo no solo en hablantes de herencia, sino también en varios nativo-hablantes del español (HN), además de los no-nativos (HL2). No obstante, a pesar de esta diversidad innegable, los programas de lengua no están al alcance con estos cambios demográficos en cuestiones curriculares ni metodológicas. En muchas universidades se sigue con el modelo tradicional que es de talla única, lo cual significa que el contenido, el proceso y el ritmo de la instrucción son uniformes para todos los estudiantes matriculados (Potowski 2002). Este modelo, además, asume que los aprendices tienen un dominio parejo de la lengua meta y que los instructores entienden las necesidades lingüísticas de estos estudiantes. Como tal, ese modelo peca de simplista, puesto que las investigaciones sobre el español como lengua de herencia en los EE.UU. han demostrado repetidas veces que los estudiantes de herencia hispana y los hablantes del español como lengua extranjera (L2) tienen características y necesidades pedagógicas diferentes (Carreira \& Hitchins Chik 2018, Potowski \& Carreira 2004). El propósito de este trabajo es ofrecer una vista panorámica sobre los desafíos que enfrenta el estudiante universitario de herencia hispana en los EE.UU. en programas de lengua corrientes.

En los apartados que siguen nos proponemos los siguientes objetivos. En el segundo apartado exploraremos más a fondo el contexto socio-cultural que rodea el tema de los HHH en los EE.UU. El tercer apartado resumirá las características de los programas de lengua universitarios para los HHH en los EE.UU. En el cuarto, se contrastarán las necesidades pedagógicas de los HHH con las de los HL2, motivando así la necesidad de una metodología y trato diferentes de los que requieren los estudiantes del español como lengua extranjera. El quinto apartado delineará los desafíos que enfrentan los $\mathrm{HHH}$ en programas de lengua española existentes. Por último, el sexto apartado resume algunas soluciones.

\section{El contexto socio-cultural}

En el momento actual, los hispanos son el grupo minoritario más grande en los EE.UU., dado que representan el 18\% de la población total del país (Oficina del Censo de los EE.UU. 2019). Además de ser el 
más grande, también es el grupo minoritario con la tasa de crecimiento más alta. Solo en la última década la población hispana en los EE.UU. ha marcado un aumento sustancial de 43\% (Ennis et al. 2011). Y aunque algunos estados tienen un porcentaje más alto de hispanos que otros, el aumento se ha observado en todos. Se estima que para el año 2060 los hispanos representarán el 29\% de la población total (Colby \& Ortman 2014). Entonces no es sorprendente que, después del inglés, el español sea la lengua más hablada en los EE.UU., con 41 millón de hablantes (Fernández Vítores 2019). Muchos de sus hablantes se categorizan como hablantes de herencia. Las definiciones de este concepto son abundantes y controvertidas, centrándose típicamente alrededor de dos parámetros: la afiliación cultural y/o la competencia lingüística. Para los propósitos de este trabajo, siguiendo a Beaudrie y Fairclough (2012), definimos al hablante de herencia como al individuo con una conexión personal o familiar a la lengua no mayoritaria.

Adicionalmente, de la población general, los hispanos son el grupo con el porcentaje más alto de la población de edad escolar. Consecuentemente, la misma tendencia de crecimiento se ha observado en la educación postsecundaria. Específicamente, a nivel nacional, la matriculación de estudiantes hispanos en universidades públicas y privadas casi se triplicó de 1.3 millones en el 1999 a 3.6 millones en el 2016 (Gramlich 2017), o de 22\% a 36\% (McFarland et al. 2019). En el 2017, en 523 instituciones postsecundarias un 25\% del estudiantado eran hispanos. Por lo general estas instituciones se concentran en ambientes urbanos y en regiones geográficas donde los hispanos han predominado tradicionalmente, como California, Tejas, Florida, Nueva York, Illinois, Nuevo Méjico y Puerto Rico.

Estos datos demográficos señalan que el perfil prototípico del estudiante universitario está en proceso de evolución. Si una de las maneras de medir el valor de la educación es a través de la medida en la que ésta responde a las necesidades estudiantiles, la pregunta que surge es cuán beneficioso es el sistema corriente para el estudiante de herencia hispana. Parece que muy poco, puesto que el índice de graduación de programas postsecundarios para los estudiantes hispanos es 10\% más bajo que para los estudiantes de descendencia anglosajona (De Brey et al. 2019), a pesar del aumento sustancial en la matriculación de los hispanos. Danini Cortéz (2014), cuyo trabajo se centra en programas postsecundarios en Tejas, señala que las barreras que enfrenta el estudiante hispano no tienen nada que ver con su etnicidad, el nivel económico de su familia o cuál fue la primera lengua que adquirieron. Al 
contrario, las barreras las crean el desequilibrio y la deficiencia ubicuos en sistemas educativos norteamericanos.

\section{Programas de lengua española existentes}

Los programas de lengua española en universidades norteamericanas no son una excepción a dicho desequilibrio y deficiencia. En muchas universidades se opta por no establecer programas particulares para los estudiantes de herencia hispana, a pesar de que las investigaciones en el área del español como lengua de herencia en los EE.UU han demostrado repetidamente que los programas de español como L2 no representan el ambiente óptimo para los HHH (Potowski 2002). Según Ingold et al. (2002), el obstáculo principal para desarrollar un programa o una vía particular para los $\mathrm{HHH}$ es la falta de interés por parte de la administración, los estudiantes y la facultad. Sin embargo, Beaudrie (2011) ha demostrado la existencia de una relación directa positiva entre la existencia de programas de lengua diseñados para los HHH y la cantidad de estudiantes hispanos en ciertas universidades.

Además, las universidades difieren en la estructura de los programas de lengua que ofrecen a los HHH. Existen programas relativamente elaborados con amplias ofertas de cursos que se diferencian entre sí por su nivel y los objetivos y destrezas lingüísticas que trabajan (p.ej. la Universidad de Arizona tiene seis cursos diferentes para los $\mathrm{HHH}$, de niveles básico, intermedio y avanzado, y se enfocan, en medidas diferentes, en destrezas lingüísticas productivas o receptivas, en registros profesionales y académicos, diferentes géneros narrativos, e inclusive en la dicción y aspectos fonéticos). Aunque el cambio se nota paulatinamente, los programas más comprensivos todavía son una excepción. El modelo predominante son los programas que consisten en uno o dos cursos que extrapolan a los estudiantes de herencia hispana del currículo general (p. e. en la Universidad de Texas A\&M la oferta para los HHH consiste en un curso de español intermedio y otro de gramática avanzada). Independientemente del nivel de sofisticación del programa, frecuentemente hay una discrepancia entre lo que ofrece el programa para los $\mathrm{HHH}$ y las expectativas de los estudiantes mismos, como, por ejemplo, promover las conciencia intracultural en oposición a promover el sentido de la identidad personal y orgullo cultural, respectivamente (DeFeo 2015).

Más importante todavía, a pesar de la presencia más marcada de los $\mathrm{HHH}$ en los programas de lengua a nivel postsecundario, todavía no 
existe una metodología acordada para enseñar el español a los HHH. Como consecuencia, los programas existentes frecuentemente carecen de objetivos pedagógicos, lineamientos curriculares, procedimientos para colocar a los estudiantes en el nivel adecuado según su competencia lingüística, instructores entrenados en cómo enseñar el español a hispanohablantes y manuales didácticos.

En el momento actual, esta brecha se soluciona de dos maneras. Una de ellas, y la más prevalente, son las clases híbridas o clases mixtas. Bajo este modelo, el espacio instructivo es compartido por los $\mathrm{HHH}$ y los HL2. En estas clases típicamente se usa la metodología del español como lengua extranjera (ELE). Adicionalmente, inclusive cuando los instructores tienen conocimiento de las características de los $\mathrm{HHH}$, raras veces están familiarizados con cómo enfrentar la dinámica de una clase mixta (Carreira \& Hitchins Chik 2018).

Otra solución son cursos particularmente diseñados para los hablantes de herencia. Estos cursos, cuyo objetivo es mejorar las destrezas escritas y la expansión de repertorios lingüísticos, con frecuencia sirven como un terreno fértil para promover la lengua estándar o para imponer el prestigio de una variedad académica idealizada, recalcando así las jerarquías lingüísticas y, a su vez, potencialmente afectando la autoestima lingüística de los estudiantes y el mantenimiento del español (Leeman 2005, Martínez 2003, Villa 1996).

\section{Las necesidades pedagógicas de los $\mathrm{HHH}$ en programas de español universitarios}

A pesar del perfil único de los estudiantes de herencia desde el punto de vista lingüístico, sociocultural y afectivo, la enseñanza de lenguas extranjeras como campo de estudio a duras penas está empezando a entender a los HHH a través de o en relación con los HL2 (Carreira \& Kagan 2011, Felix 2009). Las investigaciones sobre el español como lengua de herencia en los EE.UU. insisten en que los estudiantes de herencia hispana y los hablantes del español como lengua L2 tienen características y necesidades pedagógicas que varían en función de dos parámetros: el lingüístico y el socio-afectivo.

Desde el punto de vista lingüístico, Carreira y Hitchins Chik (2018) destacan que en el ambiente instructivo los HHH tienen puntos fuertes y carencias diferentes comparado con los HL2. En las columnas a continuación se representan los puntos fuertes de los HHH y los HL2, respectivamente, según las autoras. 


\section{HHH}

Hablar

Escuchar

Uso espontáneo de la lengua

Módulo interpersonal

Conocimiento implícito de la lengua

Lengua coloquial
HL2

Escribir

Leer

Uso ensayado de la lengua

Módulo presentacional

Conocimiento explícito de la lengua

Lengua formal

Por lo tanto, las necesidades lingüísticas de estos estudiantes en programas de lengua son diferentes también. Las autoras las resumen de la siguiente manera:

\section{HHH}

Escribir

Leer

Uso ensayado de la lengua

Módulo presentacional

Conocimiento explícito de la lengua

Lengua formal

\begin{abstract}
HL2
Hablar

Escuchar

Uso espontáneo de la lengua

Modulo interpersonal

Conocimiento implícito de la lengua

Lengua coloquial
\end{abstract}

Entonces, desde el punto de vista lingüístico, se puede notar que tanto las relativas habilidades como las necesidades pedagógicas de los HHH son directamente inversas a las de los HL2.

En cuanto al aspecto socio-afectivo del aprendizaje de la lengua meta, los HHH atraviesan dos mundos lingüísticos y culturales y estudian la lengua y la cultura meta para poder entender su propia identidad y para poder establecer conexiones o fortalecer las relaciones existentes con otros hablantes de la lengua, en particular, con familiares y amigos en los EE.UU y en el extranjero. Además, con mucha frecuencia enfrentan el rechazo y otros desafíos particulares de ser bilingüe y bicultural. Sobra decir que ninguna de estas características socio-afectivas se observan en los HL2 (es decir, no son bilingües ni biculturales, no tienen lazos familiares con la lengua y cultura meta, ni se identifican en términos de la lengua y cultura meta).

Adicionalmente, Hedgcock y Lefkowitz (2016) analizaron las percepciones de los HHH sobre su propia competencia lingüística, sus necesidades y hábitos de uso lingüístico y cómo éstos los señalan como usuarios diferentes en comparación con angloparlantes, aprendices del español como L2. Los autores concluyen que las variables socio-afectivas que separan a los HHH de los HL2 son las actitudes hacia el español, los 
prejuicios sobre las normas lingüísticas, los esfuerzos de los aprendices para obtener legitimidad como usuarios del español y los obstáculos en la interacción, como la ansiedad y la cohibición social. En otras palabras, la imagen que tienen sobre sí mismos estos estudiantes es una imagen de usuarios inseguros del español, con muchas caracterizaciones negativas, paralelas a las percepciones de sus instructores y sus compañeros nonativos del español.

\section{Los desafíos del sistema corriente}

La discrepancia entre la particularidad del perfil de los $\mathrm{HHH}$ y la realidad que enfrentan en el espacio instructivo en programas de lengua española existentes crea una serie de desafíos para estos estudiantes, puesto que refuerzan los estereotipos sobre su estatus en la sociedad y el estigma relacionado con su variedad lingüística del español. A continuación se resumen los desafíos más frecuentes:

1. Las expectativas estudiantiles no coinciden con los objetivos y el contenido del curso, puesto que no existe un estándar metodológico.

DeFeo (2015) estudió las percepciones de los hablantes de herencia hispana en una clase de español como lengua extranjera para principiantes. A pesar de sus identidades que claramente estaban vinculadas a los EE.UU. y el español fronterizo, los participantes del estudio percibían que el currículo se enfocaba en el español de España y el resto de los países extranjeros de habla hispana, pero que generalmente se omitía la mención del español estadounidense. Sus interpretaciones del currículo se contradecían con sus expectativas del mismo. Es decir, interpretaban que el español sirve para viajar, aunque ellos lo utilizaban rutinariamente en aplicaciones locales; que la cultura española existe en otros países, aunque ellos tenían conexiones profundas con la familia y la comunidad en los EE.UU.; y que el español castellano era la variedad ideal, aunque sus propias identidades bilingües estaban ancladas al español estadounidense. Por sus mensajes discrepantes, los estudiantes de herencia hispana suelen percibir estos programas como inauténticos, inaplicables y sin alineamiento con sus identidades.

2. Las variedades lingüísticas de los estudiantes de herencia hispana siguen siendo variedades despreciadas.

A pesar del aumento sustancial de estudiantes de herencia hispana en programas de lengua española en los EE.UU., las variedades regionales habladas por estos alumnos siguen siendo despreciadas (Potowski \& Carreira 2004). Potowski y Carreira (2004) condujeron un estudio sobre 
el estándar nacional de la enseñanza del español como lengua de herencia en el cual investigaron el desarrollo de los maestros en estos programas. Concluyeron que la mayoría de los alumnos se sentían frustrados con sus instructores de lengua, porque estos tenían expectativas demasiado altas. En este estudio, los instructores se enfocaban solamente en los errores de los alumnos en vez de fortalecer los aspectos de la lengua que ya tenían en su repertorio lingüístico. Además, la corrección de errores se centraba principalmente en las características específicas de su propia variedad de la lengua más que en los errores lingüísticos que resultan de una adquisición incompleta. Es decir, en vez de enfocarse en aspectos que les ayudarían a los alumnos de herencia a expandir su repertorio lingüístico, el enfoque del aprendizaje consistía en una comparación entre la variedad «correcta» (estándar) del español contra la variedad «incorrecta» (subestándar). Como resultado, estos estudiantes percibían que su manera de hablar era inferior porque no era lo «correcto»o «nativo».

Estos enfoques pedagógicos que se centran en la idea de lo correcto (vs. lo incorrecto; ing. correctness) y en el proceso de corregir, es decir, la corrección misma (ing. correction) promueven el bilingüismo sustractivo. Este se refiere a la situación en la cual la lengua mayoritaria se aprende a costa de la minoritaria y gradualmente la reemplaza. Su resultado inmediato es que los estudiantes desarrollan perspectivas negativas sobre su lengua y cultura, lo cual, a su vez, favorece y facilita el desplazamiento lingüístico en grupos o culturas minoritarias tras un intento de unirse a la cultura dominante. Bayram et al. (2016) señalan que problematizar las gramáticas de los $\mathrm{HHH}$, además de no estar basado en hallazgos científicos, es contraproducente desde el punto de vista pedagógico, porque afecta de manera negativa el perfil afectivo del estudiante, conduciendo a la ansiedad y la autoestima lingüística baja, además de eliminar la motivación para continuar con los estudios de la lengua de herencia.

3. La inexistencia de un estándar de preparación para instructores tiene como consecuencia la discriminación interna.

Hasta en las instituciones con programas para los $\mathrm{HHH}$, la mayoría de los instructores no reciben la formación apropiada para dirigir una clase de español como lengua de herencia (Potowski \& Carreira 2004). Las autoras destacan que es erróneo suponer que los instructores capacitados solamente en la enseñanza de L2 son capacitados para enseñar cursos de herencia. En ese caso, a menos que los maestros reciban suficiente formación para tratar con los alumnos de herencia, la colocación de estos estudiantes en clases de español para no-nativos seguirá siendo ineficaz y su éxito inexistente (Beaudrie 2011). 
Intrínsecamente relacionada con la falta de preparación adecuada de los instructores es la discriminación lingüística interna. Valdés et al. (2003) advierten que como instructores de español, directa o indirectamente, transmitimos ideologías implícitas sobre:

a) el nacionalismo, señalando que una lengua equivale a una nación,

b) el estándar, comprometiéndonos con la pureza lingüística y la idea del habla correcta, y

c) el monolingüismo idealizado, indicando la superioridad del hablante monolingüe educado.

Billsy Vigil (2008) también demostraron la existencia de una relación directa entre la discriminación lingüística interna y el desplazamiento lingüístico. En particular, concluyeron que los hablantes de español suelen discriminar contra las variedades regionales del español hablado en los EE.UU y expresan actitudes negativas hacia prácticas bilingües típicas como el cambio de códigos y el uso de préstamos. Los hallazgos de Román et al. (2019) coinciden con los de Bills y Vigil (2008). Además, su análisis de actitudes lingüísticas de los instructores en escuelas bilingües en el norte de Tejas, también reporta que, basándose en la variedad del español de sus estudiantes, los instructores sin preparación adecuada acostumbraban sacar conclusiones precipitadas sobre el estatus socioeconómico de los padres, su nivel de educación y su habilidad de mantener a sus hijos. El mismo estudio también señaló que estos instructores tenían la tendencia de aceptar las variedades del español de sus compañeros de trabajo, mientras discriminaban contra las de los estudiantes y sus padres. También solían atribuir el uso del cambio de códigos en los adultos a una preferencia personal, mientras que el de los estudiantes lo atribuían a la falta de conocimiento de lengua meta. Más de $80 \%$ de los instructores en este estudio reportaron la falta de familiaridad con temas pertinentes al bilingüismo en los EE.UU, la variación lingüística en el mundo hispanohablante y temas sociolingüísticos. Como resultado, la corrección predomina en las filosofías docentes de estos instructores (Correa 2011).

\section{Contemplando soluciones}

Pese a que nuestro entendimiento de la complejidad de los procesos que subyacen el bilingüismo ha mejorado y a la explosión de las investigaciones sobre el español en los EE.UU., los programas de lengua española para los $\mathrm{HHH}$ en ambientes universitarios estadounidenses siguen siendo un terreno fértil para la discriminación de los mismos 
estudiantes para los que fueron creados, ya que típicamente reflejan ideologías monolingües y fomentan perspectivas monoglósicas, dejando a los estudiantes enajenados y marginalizados.

Una manera de combatir dicha situación sería la adopción de enfoques que fomentan el bilingüismo aditivo. Este se refiere a la situación en la cual el individuo o el grupo aprenden la lengua mayoritaria sin impactos negativos en la lengua minoritaria. Bajo este acercamiento, la lengua mayoritaria añade valor, agrega, en vez de reemplazar la minoritaria. Los enfoques pedagógicos que fomentan el bilingüismo aditivo están centrados en la idea de contextos o registros apropiados (ing. context / register apropriatness) y la expansión de repertorios lingüísticos (Cantero García 2014).

Sin embargo, algunos expertos (véase p.ej. Leeman 2005, Lynch 2012, Holguín Mendoza 2018, etc.) señalan que los enfoques basados en la expansión de repertorios lingüísticos, aunque mejores que aquellos que promueven la erradicación de la lengua minoritaria, todavía recalcan las jerarquías lingüísticas y favorecen una variedad académica idealizada. Las clases de lengua para los HHH basadas en estos acercamientos están fundadas en ideologías lingüísticas que se anclan en un discurso elitista, cuyo objetivo es excluir. Suponen, además, que las destrezas lingüísticas de los HHH son deficientes (Jaffe 2007). Ruiz (1984) aboga por que se abandone la conceptualización de la lengua de los $\mathrm{HHH}$ como «un problema» y sugiere la interpretación de la lengua como un recurso. Lynch (2012) y Leeman (2005) coinciden con la postura de Ruiz (1984) y destacan que el problema crucial con estos enfoques proviene de su interpretación de la lengua. Específicamente, la perciben como un sistema fijo que puede adquirirse hasta cierto grado culminante, en vez de asumir una perspectiva dinámica, según la cual la lengua es una serie de prácticas.

Adicionalmente, las investigaciones han demostrado el rol crucial de la identidad en la adquisición de lenguas y su mantenimiento. Los hablantes de L2 y de herencia que desarrollan identidades positivas con la lengua tienen oportunidades más altas de adquirirla y mantenerla (Fishman 2006). Por otro lado, las inseguridades lingüísticas impiden el desarrollo lingüístico (Martínez 2003).

Siguiendo esa línea de pensamiento, las investigaciones han señalado una demanda fuerte para implementar pedagogías lingüísticas críticas, que de manera eficiente tratan los múltiples desafíos lingüísticos y sociales que enfrentan los $\mathrm{HHH}$ en los programas de lengua en los EE.UU. Específicamente, las pedagogías críticas son un conjunto de 
prácticas pedagógicas que requieren que el estudiante mejore su conciencia lingüística para poder descomponer y cuestionar las jerarquías sociopolíticas, la dominación y las creencias y prácticas que las generan (Leeman 2005). Desde el punto de vista lingüístico, se trata de proponer programas centrados en desarrollar la conciencia lingüística crítica (ing. critical language awareness), cuyo objetivo es empoderar a los estudiantes, lograr que sean conscientes de sus propias ideologías lingüísticas y que entiendan los significados sociales de diferentes variedades lingüísticas en sus comunidades de práctica. Estos enfoques típicamente se basan en conceptos como la agencia (filosófica), la acción y la identidad (Leeman 2005). También incorporan temas sociolingüísticos y pragmáticos. Su meta primordial es validar las variedades lingüísticas de los estudiantes HHH y, a su vez, sus identidades.

Además, estos acercamientos críticos a programas de lengua diseñados para los $\mathrm{HHH}$ asumen que la competencia lingüística que los HHH traen de sus casas y sus comunidades son una ventaja y un conocimiento cultural relevante. Holguín Mendoza (2018) destaca que el estudiante $\mathrm{HHH}$ debe decidir si utilizará o no ciertos rasgos lingüísticos, no basándose en el miedo y la estigmatización, sino en su propia experiencia y objetivos lingüísticos, navegando las intersecciones de sus propias prácticas como hablante de herencia y aquellas más frecuentemente usadas en entornos académicos y profesionales. De esta manera se les prepara a los $\mathrm{HHH}$ a tomar decisiones informadas sobre su uso lingüístico. El objetivo es desarrollar en los HHH la auto-estima y el orgullo por el conocimiento lingüístico que ya poseen (Holguín Mendoza 2018).

Lograr esta meta es virtualmente imposible sin instructores con entrenamiento en la variación lingüística, el bilingüismo o el contacto de lenguas (Román et al. 2019), dado que estos son los profesionales más adecuados para informar a los HHH de la validez de sus variedades y ayudarles a desarrollar dicha conciencia lingüística crítica, además de ayudarles a expandir sus habilidades lingüísticas.

No obstante, Beaudrie (2015) ha encontrado que a pesar del aumento de enfoques que se basan en la apreciación de diferentes variedades lingüísticas comparado con los enfoques basados en su erradicación, todavía la adopción de las prácticas pedagógicas sociolingüísticamente conscientes ha sido lenta y la enseñanza del estándar sigue siendo el modelo central en muchos programas de HHH. 


\section{Conclusión}

Frente a la ausencia de programas diseñados específicamente para los $\mathrm{HHH}$, estos estudiantes típicamente acaban en clases híbridas, con hablantes no nativos y a veces nativos del español, donde el método de enseñanza es de talla única para todos los estudiantes matriculados y se basa en enfoques pedagógicos diseñados para la adquisición del español como L2. Inclusive cuando están en clases diseñadas específicamente para los $\mathrm{HHH}$, se trata con frecuencia de clases que marginalizan las prácticas lingüísticas no dominantes, marginalizando así a los mismos estudiantes para los cuales estas prácticas forman elementos centrales de sus repertorios comunicativos.

Este trabajo argumenta que los programas de lengua para los HHH han de ser mucho más que solo un espacio en el que se explora la competencia lingüística de los estudiantes matriculados. Entender mejor la complejidad que subyace entre la lengua y la identidad en contextos multilingües como el de los HHH en los EE.UU. significa reconocer que la lengua está intrínsecamente relacionada con relaciones de poder, estructuras sociales, identidades de los hablantes e ideologías lingüísticas. $\mathrm{Si}$ los programas de lenguas universitarios en los EE.UU. quieren ser eficientes y acoplarse al prototipo nuevo del estudiante universitario, deben incorporar estos aspectos.

\section{REFERENCIAS BIBLIOGRÁFICAS}

Bayram 2016: Faith Bayram et al. «Why should formal linguistic approaches to heritage language acquisition be linked to heritage language pedagogies?». Peter P. Trifonas \& Themistoklis Aravossitas (eds.), Handbook of Research and Practice in Heritage Language Education, Cham: Springer, 187-205.

Beaudrie 2011: Sarah Beaudrie. «Spanish heritage language programs: A snapshot of current programs in the southwestern United States». Foreign Language Annals, 44: 321-337.

Beaudrie 2015: Sarah Beaudrie. «Instructional effectiveness in the SHL classroom: Comparing teacher and student perceptions». Journal of Hispanic Higher Education, 14: 274-297. [https://doi. org/10.1177/1538192715575372] Web. 15/06/2019. 
Beaudrie \& Fairclough 2012: Sarah Beaudrie \& Martha Fairclough (eds.), Spanish as a heritage language in the United States: State of the field. Georgetown: Georgetown University Press.

Bills \& Vigil 2002: Garland D. Bills \& Neddy E. Vigil. The Spanish of New Mexico and southern Colorado: A linguistic atlas. Albuquerque: University of New Mexico Press.

Cantero García 2014: Víctor Cantero García. «La mejora de la competencia lingüística desde la interacción comunicativa: Una propuesta para la enseñanza bilingüe». Didáctica: Lengua y Literatura, 26: 81-104.

Carreira \& Hitchins Chik 2018: María Carreira \& Claire Hitchins Chik. «Differentiated teaching: A primer for heritage and mixed Classes». Kim Potowski (ed.), The Routledge Handbook of Spanish as a Heritage Language, New York: Routledge, 359-375.

Carreira \& Kagan 2018: María Carreira \& Olga Kagan. «Heritage language education: A proposal for the next 50 years». Foreign Language Annals, 51: 152-168. [https://doi.org/10.1111/flan.12331] Web. 15/06/2019.

Correa 2011: Maite Correa. «Advocating for critical pedagogical approaches to teaching Spanish as a heritage language: Some considerations». Foreign Language Annals, 44: 308-320. [https:// doi.org/10.1111/j.1944-9720.2011.01132.x] Web. 15/06/2019.

Colby \& Ortman 2014: Sandra L. Colby \& Jennifer M. Ortman. «Projections of thesizeand composition of theU.S.population:2014to2060».Current Population Reports. Washington, DC: U.S. Census Bureau: P25-1143. [https://www.census.gov/content/dam/Census / library/publications / 2015 /demo/p 25 -1143.pdf] Web. 15/06/2019.

Danini Cortéz 2014: Josie Danini Cortéz. «Barriers Hispanic students face graduating from high school». IDRA Newsletter, October 2014. [http://www.idra.org/IDRA_Newsletter/] Web. 25/10/2017.

De Brey et al. 2019: Cristobal De Brey et al. «Postsecundary graduation rates». Status and Trends in the Education of Racial and Ethnic Groups 2018. Washington, DC: National Center for Educational Statistics - U.S. Department of Education.

[https://nces.ed.gov/programs/raceindicators/indicator_red.asp] Web. 01/06/2019.

DeFeo 2015: Dayna J. DeFeo. «Spanish is foreign: Heritage speakers' interpretations of the introductory Spanish language curriculum». International Multilingual Research Journal, 9: 108-124. [https:// doi.org/10.1080/19313152.2015.1016828] Web. 15/06/2019. 
Ennis et al. 2011: Sharon R. Ennis et al. «The Hispanic population: Census briefs 2010». Washington, DC: U.S. Census Bureau. [https://www.census.gov/prod/cen2010/briefs/c2010br-04.pdf] Web. 01/06/2019.

Felix 2009: Angela Felix. «The adult heritage Spanish speaker in the foreign language classroom: a phenomenography». International Journal of Qualitative Studies in Education, 22: 145-162.

Fernández Vítores 2019: David Fernández Vítores. «El español una lengua viva: Informe 2019». Madrid: Instituto Cervantes.

[https://cvc.cervantes.es/lengua/espanol_lengua_viva/] Web. 01/06/2019.

Fishman 2006: Joshua A. Fishman. «Three hundred-plus years of heritage language education in the United States». Guadalupe Valdés, Joshua A. Fishman, Rebecca Chávez y William Pérez (eds.), Developing Minority Language Resources: The Case of Spanish in California, Clevedon: Multilingual Matters, 12-23.

Gramlich 2017: John Gramlich. «Hispanic dropout rate hits new low, college enrollment at new high». Washington, DC: Pew Research Center. [http://pewrsr.ch/2x2wyy1] Web. 01/06/2019.

Holguín Mendoza 2018: Claudia Holguín Mendoza. «Critical language awareness (CLA) for Spanish heritage language programs: Implementing a complete curriculum». International Multilingual Research Journal, 12: 65-79.

[https://doi.org/10.1080/19313152.2017.1401445]

Web. 22/10/2019.

Hedgcock \& Lefkowitz 2016: John S. Hedgcock \& Natalie Lefkowitz. «Differentiating heritage and foreign language learners of Spanish: Needs, perceptions, and expectations». Applied Language Learning, 26: $1-38$.

Ingold et al. 2002: Catherine W. Ingold et al. «Report on the NFLC/ AATSP survey of Spanish language programs for native speakers». Hispania, 85: 324-329.

Jaffe 2007: Alexandra Jaffe. «Minority language movements». Monica Heller (ed.), Bilingualism: A Social Approach, London: Palgrave Press, 50-70.

Leeman 2005: Jennifer Leeman. «Engaging critical pedagogy». Foreign Language Annals, 38: 35-45.

Lynch 2012: Andrew Lynch. «Key concepts for theorizing Spanish as a heritage language». Sarah Beaudrie \& Martha Fairclough (eds.), 
Spanish as a Heritage Language in the United States: The State of the Field, Georgetown: Georgetown University Press, 79-97.

McFarland et al. 2019: Joel McFarland et al. «College enrollment rates». The Condition of Education 2019. Washington, DC: National Center for Educational Statistics - U.S. Department of Education. [https:// nces.ed.gov/programs/coe/pdf/coe_cpb.pdf] Web. 01/06/2019.

«Race and Hispanic origin estimates». Quick Facts. Washington, DC: U.S. Census Bureau, 2019.

[https://www.census.gov/quickfacts/fact/table/US/RHI725218] Web. 15/06/2019.

Ruíz 1984: Richard Ruíz. "Orientations in language planning». $N A B E$ Journal, 8/2: 15-34. [https://doi.org/10.1080/08855072.1984.10 668464] Web. 18/10/2019.

Potowski 2002: Kim Potowski. «Experiences of Spanish heritage speakers in university foreign language courses and implications for teacher training». ADFL Bulletin, 33: 35-42.

Potowski \& Carreira 2004: Kim Potowski \& María Carreira. «Towards teacher development and national standards for Spanish as a heritage language». Foreign Language Annals, 37: 421-431.

Roberts 2018: Gregg Roberts et al. «Monolingualism is the illiteracy of the twenty-first century». Hispania, 100: 116-118. [doi:10.1353/ hpn.2018.0028] Web. 16/07/2019.

Román et al. 2019: Diego Román et al. «Internal linguistic discrimination: A survey of bilingual teachers' language attitudes toward their heritage students' Spanish». Bilingual Research Journal, 42: 6-30. [https://doi.org/10.1080/15235882.2018.1563006] Web. 01/11/2019.

Ruíz 1984: Richard Ruíz. "Orientations in language planning». NABE Journal, 8/2: 15-34. [https://doi.org/10.1080/08855072.1984.10 668464] Web. 16/07/2019.

Valdés 2003: Guadalupe Valdés et al. «Language ideology: the case of Spanish in departments of foreign languages». Anthropology \& Education Quarterly, 34: 3-26.

Villa 1996: Daniel J. Villa. "Choosing a "standard" variety of Spanish for the instruction of native Spanish Speakers in the U.S.». Foreign Language Annals, 29: 191-200. 


\title{
HERITAGE SPANISH SPEAKERS \\ IN THE AMERICAN COLLEGE CLASSROOM
}

\begin{abstract}
Summary
The goal of this paper is to offer a panoramic overview of the reality that Spanish heritage learners face in a language classroom in a US college setting. According to the most recent demographic projections, in the near future Spanish heritage speakers will cease to be a minority in post-secondary institutions in the US. However, there is still no agreed-upon method to teach Spanish to heritage speakers, despite the fact that the Spanish/English contact has shaped the American landscape for centuries and research on US Spanish varieties stresses the need for methods of language teaching specifically designed for heritage speakers. This paper summarizes the challenges heritage Spanish speakers encounter in a language classroom setting as a result of this discord and discusses solutions.
\end{abstract}

Keywords: heritage speakers of Spanish, Spanish language programs, linguistic awareness, additive bilingualism, critical pedagogy. 